\title{
Personality Traits Recognition Through Twitter's Profile Picture
}

\section{Hala Ibrahim Darwish}

Lecturer of educational media/

Faculty of Specific Education/

Port-Said University
Hany Ibrahem El-Batal

Professor of educational media/

Faculty of Specific Education/

Port-Said University

Abstract

Social media is a place where users present themselves and preferences to the world by daily posting. While social networks are entrenched as a central element of everyday life, posts to social networks tend to be more positive as individuals want to portray a positive image to their friends that vary from individual to another.

The research conducted in this paper has focused on the personality of Twitter's user based on research, that aims at benchmarking emotion recognition algorithms through face reaction. Previous studies concluded that the user's account profile picture refers to self-representation. Detection, extraction, and evaluation of these facial expressions in an image are being measured by algorithms which allow for automatic recognition of the human emotion. The objective of the present paper is to infer the personality traits from the profile pictures and analyse those pictures to indicate the personality traits that the users may have intended to portrait themselves on the social media. The input data in the study is $\mathbf{5 0 0}$ twitter profile pictures by using Face++ API. The paper described the type of data collected and the analysis methods that are utilized to predict the user's personality and measured the personality through the user's account profile picture and this is what the users chose to portray themselves into. The study has expounded that Twitter profile pictures carry relevant information for classifying the personality traits of the individuals who post them based on the standard BigFive model. Therefore, the measured personalities are not necessarily the true personality. 
Online social media has grown dramatically over the last two decades and become a widespread medium for information dissemination and interaction between millions of users (Huberman, et al. 2009; Kwak, et al. 2010). Previous statistics have shown that individuals are spending more of their time on the Internet, or through Social Networking Services (SNS) (Alexa 2017). It is apparent that SNSs are data-rich platforms to study personality and human behaviors, which allows researchers to base their analyses of individuals' personality on digital records of human behavior. (Kosinski, et al. 2013).

The sheer volume of interaction between social media users has enabled many researchers to study the online user's personality. At the same time, a popular subject for interdisciplinary research, involving domains such as Computer Science (Danah, et al. 2010), Health (Lampos, Cristianini, 2012) and Psychology (Danah, et al. 2010). In 2005, a survey of social networking websites estimated that among all sites on the web, there were roughly 115 million social media members (Golbeck, 2005). In late 2010, (Seger, Miller, 2010) was estimated that Twitter had about 175 million registered users worldwide, producing about 65 million tweets per day.

On each of the social media platforms, the first impression counts, where a profile picture carries a unique meaning and attempts to convey a special message. The information included in the individual's profiles pictures, define who they are on social media in silent ways. Thus, it allowed users to make an opinion on other users on the social media platform based on a photo in a few seconds.

Twitter is one of the most popular social networking and micro-blogging or micro-sharing platform whose users publish short messages or tweets and connects a widely diverse array of people from all over the world, erasing barriers and boundaries. Furthermore, Twitter is so easily customizable and openended; it has continued to become more and more popular with individuals and companies (Laura, et al. 2009). In addition to that, Twitter has been modified by the usage of new communication technologies and offer new ways for self-presentation.

Individuals nowadays are heavily using social media to 
keep track of their daily activities and preferences and spend a considerable amount of effort in order to form and manage impressions, especially in the initial stage of social interactions. Several studies reported that some social media platforms users, such as Facebook users, engage in actively creating and modifying images of themselves by adjusting their profiles, including descriptions and pictures, and displaying their likes and dislikes (Gonzales, Hancock, 2008). Celli, found that personality could be extracted from profile pictures and interaction styles (Celli, et al. 2014).

Moreover, social psychology research has also highlighted that personality plays a vital role in the way people manage the images they convey in self-presentation (Krämer, Winter, 2008) As well as, how an individual presents himself/herself before the audience. It has been noted in (Correa, Hinsley, Zuniga, 2010) that self-presentation is mainly represented by posting status, images or videos. Many researchers have shown that there is an interaction between social media and the personality of the online user. Facial expression is the most common focused topic while predicting one's online personality through face images. Thus, some studies reported that users could make accurate personality impressions through social network user profiles (Vazire, Gosling, 2004). While, other studies have investigated the specific features from user profiles and photos that can be used to create personality impressions (Counts, Stecher, 2009). For instance, the results obtained by Hall et al. (Hall, Pennington, Lueders, 2013) indicate that researchers could accurately estimate Conscientiousness, Extraversion and Agreeableness of unknown profile owners . While, Heide Van et al. (Heide, et al. 2012) have shown that in the context of Facebook, photos may have more impact on judgments of Extraversion than textual self-disclosures.

Based on these previous findings, this paper addresses the task of predicting the impact of Twitter users based only on features under their direct control, such as profile pictures and if can be used to predict the user's personality.

Identity claims, like the capture angle, are the symbolic 
declarations that individuals make to themselves or others in an attempt to convey how they would like to be seen.

From a psychology point of view, personality is the set of the behavioral and emotional responses of an individual. A considerable amount of psychology researches has shown that facial expressions are the basis for personality detection in humans (Halder, et al. 2016). The personality of the individual is based mainly on the standard Big-Five model of personality trait. In this model, the human personality is being divided into five categories, namely Extraversion, Openness, Neuroticism, Conscientiousness, and Agreeableness (McCrae, John, 1992).

The individual's public page on Twitter, also known as an avatar. Some Twitter's users change their avatars almost daily to different photos of themselves, while other users leave their avatars the same all the time, others change theirs according to a specific occasion, holiday, themed photos for Christmas etc. However, many of the photos that an individual wants to use for his/her Twitter avatar may include other people. Alternatively, the picture composition may not allow getting a good headshot.

In this study, the authors focus on static images, especially on self-selected profile pictures from the Twitter platform. While users can post other photos, studying profile pictures are reflecting the impressions that the users want to convey to others. While (Back, et al. 2010) states that social media allows a user to shape his or her personality and idealized view searching for 'idealized virtual identity hypothesis', result shows that social media behavior usually represents an extension of one's self as an 'extended real-life hypothesis', thus allowing others to observe the users' real personality.

The individual's face is the main subject for predicting the personality from images, which includes face detection, face recognition, emotion recognition, sentiment analysis from the images. The paper focuses on the most widely-accepted trait framework in the history of personality psychology, the FiveFactor Model (FFM), or "Big Five".

This study is also related and based on research, that aims at benchmarking emotion recognition algorithms. 


\section{Literature Review}

\section{Emotion recognition based on facial expressions:}

There have been many approaches in using computers for detection, extraction, and recognition of human facial features and expressions, as this topic is of heed in many fields covering both social sciences and engineering. For instance, the American psychologist Ekman noticed that some facial expressions corresponding to certain emotions are common for all people independently of their gender, race, education, and ethnicity, etc. He proposed the discrete emotional model using six universal emotions: happiness, surprise, anger, disgust, sadness and fear (Ekman, Friesen, 1971). (Figure 1).

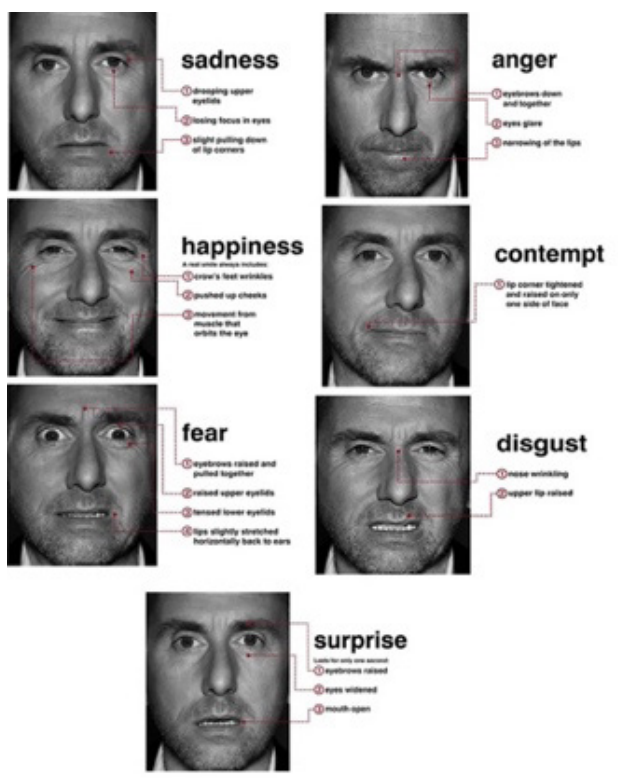

Figure 1. presents the intercorrelation of the emotion features In Ekman's Emotions Model.

Facial analysis is becoming easier to use through research. In particular, Face++ has been used in a variety of studies because of the Higher Accuracy (Jisun A. \& Ingmar W., 2016). Moreover, (Soon-Gyo J. et.al, 2018) Face++, with an accuracy rate of greater than $90 \%$, the research provides a comprehensive measurement of four widely-used face detection tools employing multiple datasets to gauge their accuracy. 


\section{The Big Five Personality model:}

In personality psychology, researchers have been interested in understanding how individuals differ. They have been trying to discover how to measure and map personality traits. Among the several theories of personality traits is the "BIG-five", or the Five-Factor Model (FFM). The model is one of the most wellresearched and well-regarded measures of personality structure in recent years and most widely used representation for automatic analysis (Alam, Riccardi, 2014).

The "Big-five" factors of personality are five broad dimensions of personality that are used to describe unique individuals (John, et al. 2010). Moreover, the model has been developed to represent as much of the variability in individuals' personalities as possible, using only a small set of trait dimensions.

The Five-Factor Model domains of personality were conceived by Tupes and Christal as the fundamental traits that emerged from analyses of previous personality tests (McCrae, John, 1992). Such extensive research has led to many psychologists to accept the Big Five as the current definitive model of personality (Schmitt, et al. 2007), (Peabody, De Raad, 2002). The Big Five model has the structure of having five components that describe the personality structure, namely "OCEAN" as follow:

- Openness to Experience. Curious, imaginative, intelligent. People with high scorers tend to be artistic appreciate diverse views, ideas, and experiences.

- Conscientiousness. Persevering, efficient, organized, responsible. Conscientious people are hard workers, extraordinarily reliable and tend to be high achievers, and planners.

- Extroversion. Active, amicable, assertive, outgoing. Friendly and energetic, extrovert people draw inspiration from social situations.

- Agreeableness. Compassionate, friendly, cooperative, nurturing. People who score high in agreeableness are optimistic, peace-keepers and trusting of others.

- Neuroticism. Anxious, sensitive and insecure. Neurotics are moody, tense, self-pitying and easily tipped into experiencing negative emotions. 
In other words, a sub-category of data focuses on facial expression recognition as mention in (Pantic, 2009), emotion recognition in (Kim, et al. 2013) and sentiment analysis in (Borth, et al. 2013; You, et al. 2015) can disclose personality traits from images. While, (Datta et al. 2006), focused on utilizing features derived from photography theory to dictate the feature that makes a picture attractive.

\section{Personality Research and Social Media:}

The word personality has come from the Latin word 'persona' which means mask, that the actors wore to change their appearance. Personality traits are the descriptions of people in terms of their relatively stable patterns of behavior, thoughts and emotions (McCrae, Costa, 2005) which make an individual unique.

On Social Media Services SMSs, users exposed to various images and videos on daily activities. With the recent rise of photo-sharing by SMS platforms (e.g., Twitter, Instagram, Pinterest, etc.), photo-posting and sharing activities on SMSs have increased vastly in popularity, making them a distinctive phenomenon in digital environments (Kim, et al. 2016).

With regard of the links between personality and posted images on social media, prior studies have been managed to predict users' personality using their Facebook profile images (Celli, et al. 2014; Segalin, et al. 2017).

Previous studies have also focused on using Twitter profiles to build predictive models and understand demographics, including psycho-demographic traits, personality, age, and gender as in (Rao, et al. 2010; Burger, et al. 2011; Schwartz, et al. 2013), others focused in using features such as social network, profile images and linguistic content (Liu, et al. 2016; Volkova, et al. 2016).

Moreover, there have been a few previous studies on how personality recognize from an appearance by humans, and it has long been a topic of concern in the field of psychology as mention in (Haxby, et al. 2000), as it has profound implications in studying personal interaction and first impressions. Some 
studies in psychology have focused on facial expressions that people commonly use as a basis for personality imputations and facial characteristics as in (Penton-Voak, et al. 2006), while other studies additionally considered the pose of the person (Naumann, et al. 2009). While computer vision algorithms have been used in some studies to automatically predict personality as a way to avoid collecting costly questionnaire data (Kamenskaya, Kukharev, 2008).

There have also been mixed results for other personality traits as shown in (Rosen, Kluemper, 2008), (Schrammel, et al. 2009), and (Selfhout, et al. 2010).

\section{Task Description and Dataset}

research questions. The mean focus of this paper is trying to answer the question: what personality trait can be inferred from the twitter's user profile picture?

The study survey data set contains (500) Twitter users profile images. The Face++ $\mathrm{API}^{*}$ has been used to predict the users' attributes and demographics. Where age and gender as either male or female information were identified. All profile images were collected on the same day for all accounts in the data set.

Image Extraction. The quality of the photo is related to beauty, attractiveness and inventiveness, which called Aesthetics. The image aspects mainly depend on the brightness, contrast and saturation. The more brightness in the image evokes creativity while darkness in the image evokes anger and sadness. For face pictures, these aspects also focus on the eyes, mouth, nose, and the face oval.

Therefore, in the present paper, the authors use public profile images as a representative for the users, which most likely, contains important psychological cues.

In order to study and interpret individuals' personalities from their profile pictures, stylistic characteristics rather than traditional computer vision features of profiles are more appropriate (Redi, et al. 2015). Most profile images contain faces, which are known to reflect personality. The study thus divided image features into two main categories: general image features 
and stylistic facial features. The former contains primary color and facial information, while the latter includes facial expressions and postures extracted from the most recognizable face from profile images.

The study used an API based on deep learning method which is Face++ for facial feature extractions. The Face ++ API along with the basic emotional expressions provides accurate face recognition to indicate demographics, face landmark (Figure 2), facial presentation as well as offering information about emotions expressed by the face detected in the profile images.

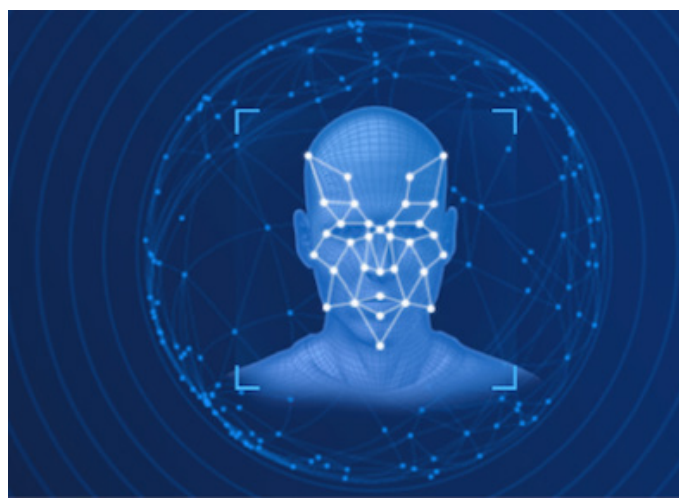

Figure 2. Presents Face

Landmarks which locate key

points of face components.

The features have been divided into the following categories:

Image Composition. A harmonious composition is an arrangement of visual elements in a photo which is essential in a work of art, and it needs to be considered to analyze an image's character as the photographers consider a large variety of elements like lines, the shape of objects, patterns, texture etc. Moreover, the color arrangement and contrast along diagonal lines, curved lines in the image have effects on artistic quality. Besides, the photographic quality influenced by the locations of the objects in the image.

Thus, the aesthetic features of basic photographic composition rules have been measured, started with the rule of thirds, where the main subject in the picture places at the border or inside an inner rectangle of a 3x3 grid, (Figure 3). Professional photos aim for simplicity, so with good quality photos, the edges are focused on the subject as well as the specific hues of a photo. 


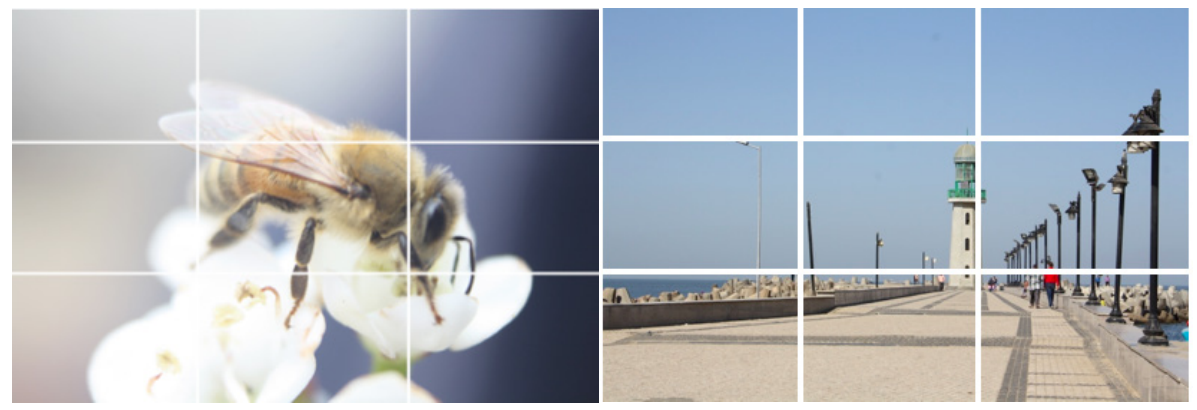

Image Type. We extract basic face-related features for each profile picture as the number of faces it contains. However, in case that there is no face in the profile image, we observe whether the user using one of the defaults Twitter profile picture images or using other people's work or stock photos.

Image Demographic. Demographic features estimated from the profile images like age, gender and race (Asian, Indian, Black or White) can also include multiple people (e.g., partner or spouse) or can use photos from their past, so that makes them appear younger or bring some memories with different people (e.g., friends, children, parents).

Image Texture. The textures in images are also important for the emotional expression of an image. In a photograph taken by a professional photographer, blur comes rare, and artists usually create sharp pictures. Blur usually happens because of glitches like poor equipment or vibration in the camera or because of the quality of the lens. However, the blur in pictures can be used efficiently to achieve the desired expression.

Lighting. The amount of light and direction of the light plays a vital role in its artistic quality. The contrast of the object also helps in emphasizing the area of interest in the photo.

Facial presentation. Which means the way a user chooses to self-present himself/herself by the profile image. Features include whether the face wears any glasses (sunglasses or prescription glasses), the closeness of the subject's face, the 3D face posture, 
which includes three angles; pitch, roll and yaw angle of the face and eye openness (Figure 4).

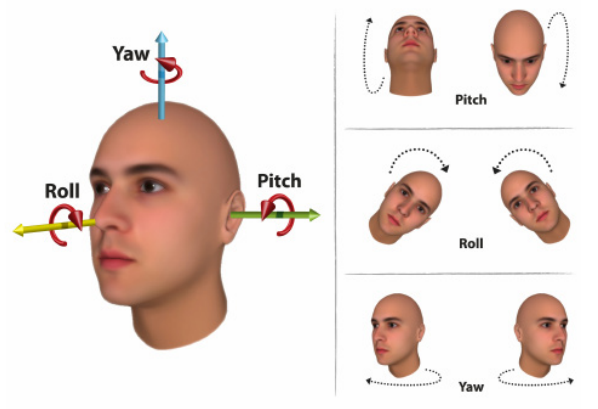

Figure 4. Orientation of the head in terms of pitch, roll, and yaw movements describing the three degrees of freedom of a human head.

Occlusion. (Figure 5) Other objects may partially occlude faces. For example, an image with a group of people, some faces may partially occlude other faces.

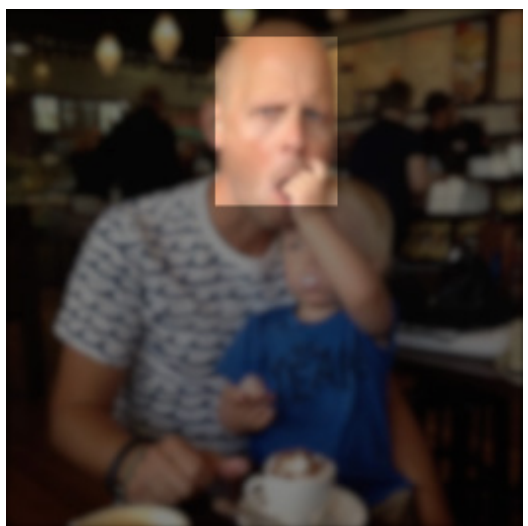

Figure 5. The Kid's face is an example of face occlusion.

Facial Expressions. The appearance of faces is directly affected by a person's facial expression and draw the attention of human observers. Although the expression of the face is fundamental in order to distinguish between the moods of a picture, algorithms that can adequately recognize the emotional expression of a human face in static images are not yet fully mature. However, the authors adopt Ekman's model of six discrete emotions: joy, sadness, anger, disgust, fear and surprise (Ekman, Friesen, 1971), were initially identified based on facial expressions. Besides, the 
Face++ API have been used to automatically extract the emotions from the detected face in each profile image as detecting frontal faces (if there are any) in the picture by using the widely available face detection algorithm by Face++ API. For each image, the number of faces found and the relative size of the most prominent face with respect to the used image. This way, we can at least distinguish pictures with people from nature, landscape or object photography, and portraits from group shots.

The six basic emotions of Ekman's model categorized into positive (joy and surprise) or negative (anger, disgust, fear and sadness). Concerning the necessary emotional expressions, Face++ also gives composite features calculated from the basic ones. Also, in this category, we add the smiling degree provided by Face+t.

Color. Colors relate to individuals' conceptual ideas like emotion and the mood and can convey rich semantics and evoke strong emotions in viewers. Some studies have shown the relation between the colors from images and psychological traits such as (Wexner, 1954) and (Verma, et al. 2018).; red with 'excitingtonic 'and 'protective-defending'; green with 'calm, peaceful and serene'; while blue indicates 'secure and comfortable' as well as peaceful.

Other Professional photographers, however, use various techniques to control colors with use specific combinations of colors that influencing the specific emotions of the viewers (Manav, 2007).

Even though colors can be used in many different ways, for analysis, the authors divided images into grayscale images and color images. For color images, the normalized red, green and blue values have taken as well as the average of the original color. Photographers take leverage of these effects on emotions to make a photograph more expressive about the story behind the photo.

Contrasts. Are regarded as another critical factor of photo quality, (Lo, et al. 2012) low contrast leads to poor quality of the photograph.

The previous study mentioned that human judgements of the 
attractiveness of images are influenced by color distributions (Huang, et 1. 2006) and aesthetic principles related to color composition (Datta, et al. 2006). Thus, brightness and contrast have been computed as the relative variations of luminance. Besides, the study represents images in the HSV (Huesaturation-Value). High saturation indicates vividness, which more appealing to the human eye. Colorfulness is calculated as the difference against grey.

The photographs captured by professionals secured higher value according to the proposed method, compared to the photographs captured by the ordinary people.

In general, (393) images have been recognized by Face++ with at least one face out of (500) profile images for the Twitter Survey data set. The missing caused by different factors; low images quality, tiny face, or not facing the camera or the face being blocked by an object. Similarly, we computed color, and image composition features only to images that were not Twitter profile default or grayscale.

\section{Analysis}

\section{Method: procedure}

The study has expounded that Twitter profile pictures carry relevant information for classifying the personality traits of the individuals who post them. Based on the standard Big- Five model, this model is a scrutinized and effective standard used for personality prediction.

The face is the index of mind, and it is unique in various ways as it is an outstanding visual object category for human (Bruce, Young, 2012). It is a complex multidimensional structure and needs a proper computing technique for recognition. While using the automatic system for face recognition, computers are easily confused by changes in illumination, variation in poses and change in angles of faces.

The personality traits in this paper have been measured following these three steps: 1-The detection, extraction, and evaluation of thefacial expressions in an image are being measured by algorithms of the Face++ API 
which allow for automatic recognition of the human emotion. 2- Using the results in the light of the discrete emotional model of the six universal emotions: happiness, surprise, anger, disgust, sadness and fear of Ekman's Emotions Model. The personal emotion is determined as shown in Figure 1.

3- The personality traits are inferred from the emotion using the conceptions of Robert R. McCrae and Oliver P. John (1992)."

Data analysis was carried out through SPSS package programs. The T-Test and One-Way ANOVA were made use of in the evaluation of data and Pearson correlation.

In these methodologies, computers have focused on detecting and recognizing features and traits of individuals such as the nose, head outline, eyes, mouth and describing a face shape and model by the size, position, and relations between these traits and features. Several researchers like (Sharif, et al. 2011) have noticed that the recognition rate of faces is high if $3 \mathrm{D}$ faces are used.

Other studies mentioned that some factors that could affect the look of an individual. These factors divided into two categories, intrinsic and extrinsic factors. (Gong, et al. 2000) Intrinsic factors include the physical condition of the human face, e.g. aging, facial expressions etc. affecting the system While extrinsic factors are those factors that become a reason to change the appearance of the face, e.g. lightening condition or pose variation.

In this section, the authors explore the correlations between personality measured through posted image features introduced in the previous section. Also, tried to investigate the main research questions, do profile pictures contain information about the user's personality?

This study organized in two tasks: firstly, we perform a correlation analysis between image features and personality scores. Secondly, we employ learning approaches to infer personality traits, given the features extracted from profile pictures.

Participants were 500 OSN users (ages 17-52 years) from the most popular social media platform (Twitter; $\mathrm{N}=324$ male, 176 female). 
Face++ API have analyzed Posted profile image from Twitter accounts in order to predict user's attributes, and demographics form their images. The Face++ API prediction methods successfully predicted a wide range of traits including age, gender, ethics impact and personality (Table 1). Besides, Face++ automatically analyses six Ekman's emotions: joy, disgust, surprise, sadness, anger, fear, as well as neutral.

Table 1 Pearson correlations between the Big Five personality traits assessed with image-predicted personality on the users.

\begin{tabular}{|c|c|c|c|c|c|c|c|}
\hline & gender & Age & Ex & Ag & Op & Ne & Co \\
\hline gander & 1 & .196 & .188 & .083 & -.015 & .118 & .106 \\
\hline Age & .196 & 1 & -.185 & -.190 & -.033 & .137 & -.077 \\
\hline $\mathrm{Ex}$ & -.188 & .185 & 1 & -.727 & -.191 & -.183 & -.148 \\
\hline $\mathrm{Ag}$ & .083 & -.190 & -.727 & 1 & -.137 & -.166 & -.147 \\
\hline $\mathrm{Op}$ & -.015 & -.033 & -.191 & -.137 & 1 & -.051 & -.003 \\
\hline $\mathrm{Ne}$ & .118 & .137 & -.183 & -.166 & -.051 & 1 & -.015 \\
\hline Con & .106 & -.077 & -.148 & -.147 & -.003 & -.015 & 1 \\
\hline \multicolumn{7}{|r}{} \\
\hline
\end{tabular}

A sample screenshot from Face++ API is shown in Figures 6, 7 and 8 .
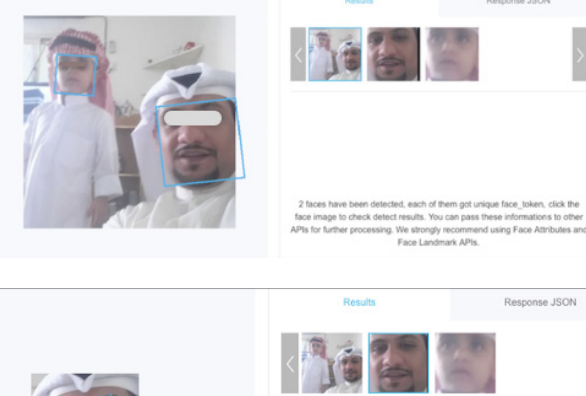

$\operatorname{sen}$

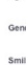

Gender
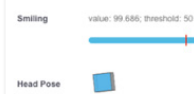

Figure 6. The number of recognized faces, Face and eyes areas are marked with a square shape.

Figure 7. there is a normalized face dotted in blue provided as well as estimation on the face rotation, estimating age, gender, head pose and the degree of the emotion. 


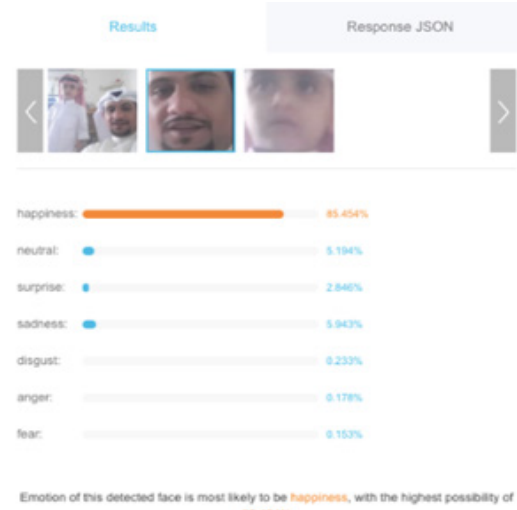

Figure 8. the recognized face

with emotions recognitions.

The analysis of Face++ API interface highlights the most critical issues in emotion recognition from facial expressions: recognition of expressions on normalized face image, recognition of face in the whole available scene, recognition of eyes within the face, and the face angle towards the camera.

Using Face++ API to analyze the pictures. The correct face recognition approach should have good pose tolerance and the capability to recognize different poses. However, standard face images-based recognition systems are likely to mistake some areas of the background as a face, shown in (Figure 9), and change the geometry of the faces and, if there is minor variation in the image, it becomes difficult for the system to recognize the face.
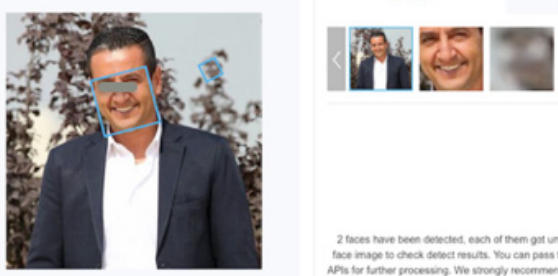

Figure 9. the system

mistakes some areas of

the background as a face.

Personality. We use the method developed by (Schwartz, et al. 2013) to assign each user scores for personality from the communal five-factor model of personality that subcategorize to five dimensions: Neuroticism, Extraversion, Conscientiousness, Agreeableness and Openness to experience. 
The (500) Twitter user's images have been organized on a web interface (Table 2); we did not investigate whether personality types can also be predicted from the banner picture or pictures posted on the individual's wall. The profiles saved onto a disc before making any mention of Twitter to the participants, to ensure that participants did not alter their Twitter profiles as a result of being in the study.

\begin{tabular}{|c|c|c|c|c|c|c|c|}
\hline \multicolumn{3}{|c|}{ Table 2 The face recognition's results by Face ++ API } \\
\hline \multicolumn{3}{|c|}{ With face } & \multicolumn{4}{c|}{ With no-face } \\
\hline One face & \multicolumn{2}{|c|}{ Multi faces } & Twitter Profile & \multicolumn{2}{c|}{ Stocked } \\
\hline Frequency & Percent & Frequency & Percent & Frequency & Percent & Frequency & Percent \\
\hline 319 & $\% 63.8$ & 74 & $\% 14.8$ & 12 & $\% 2.4$ & 95 & $\% 19$ \\
\hline
\end{tabular}

We computed univariate Pearson correlations between each visual feature and personality score. Demographic traits; age and gender are known to affect both personality features as mentioned in (McCrae, et al. 1989). The study has been used partial correlation for gender and age (predicted from a users' profile picture by Face++ API). We also include correlations with age and gender separately in (Table 3 ) in order to highlight the relevant patterns caused by demographic factors. Besides, we show a single average correlation for 'Color Emotions' and 'Rule of Thirds' which presented in (Table 4).

Table 3 the approach for predicting user demographics

\begin{tabular}{ccc}
\hline & Frequency & Percent \\
\cline { 2 - 3 } male & 324 & $\% 64.8$ \\
Female & 176 & $\% 35.2$ \\
sum & 500 & $\% 100$ \\
\hline
\end{tabular}

Table 4 Pearson correlations between profile image and Big Five personality controlled with age and gender.

\begin{tabular}{cccccccc}
\hline Feature & \multicolumn{2}{c}{ Demographics } & \multicolumn{5}{c}{ Personality Trait } \\
\hline color & gender & Age & $\mathrm{Ex}$ & $\mathrm{Ag}$ & $\mathrm{Op}$ & $\mathrm{Ne}$ & $\mathrm{Co}$ \\
\hline Grayscale & .002 & -.079 & -.033 & .023 & .069 & .012 & -.005 \\
Red & .012 & .117 & .076 & .062 & -.064 & -.016 & .010 \\
Green & .013 & .129 & .084 & -.074 & -.059 & -.010 & .006 \\
Blue & .012 & .117 & .076 & -.061 & -.064 & -.016 & .011
\end{tabular}




\begin{tabular}{cccccccc} 
Brightness & -.023 & .018 & .092 & .049 & -.030 & -.031 & .047 \\
Contrast & .020 & .027 & -.022 & -.014 & .064 & .027 & .021 \\
Saturation & .025 & .041 & -.057 & -.064 & .063 & .024 & .040 \\
Blur & .011 & .000 & -.052 & .058 & .011 & -.031 & .006 \\
Hue & -.046 & -.049 & .042 & .044 & -.165 & .017 & .005 \\
Naturalness & .018 & .087 & .103 & -.032 & -.115 & -.003 & .041 \\
Sharpness & -.013 & -.004 & .022 & -.042 & .013 & .024 & -.020 \\
\hline Image & gender & Age & Ex & $\mathrm{Ag}$ & $\mathrm{Op}$ & $\mathrm{Ne}$ & $\mathrm{Co}$
\end{tabular}

Composition

\begin{tabular}{cccccccc}
\hline Rule of Third & -.013 & .052 & .037 & .055 & -.002 & -.022 & -.014 \\
Edge & -.032 & .048 & -.005 & .000 & -.028 & .012 & .010
\end{tabular}

Distribution

\begin{tabular}{cccccccc} 
** ruleofthird33 & -.013 & .091 & -.044 & .012 & .009 & .024 & .022 \\
background & .027 & .075 & -.049 & .032 & -.009 & .017 & .016 \\
Hue Count & -.032 & .048 & -.005 & .000 & -.028 & .012 & .010 \\
\hline Image Type & gender & Age & $\mathrm{Ex}$ & $\mathrm{Ag}$ & $\mathrm{Op}$ & $\mathrm{Ne}$ & $\mathrm{Co}$ \\
\hline No-face & 0.063 &. & .094 & 0.16 & .32 & .021 & 0.34 \\
Default Image &. &. & -.034 & 0.016 & .022 & .021 &. \\
One Face & .045 & .117 & .051 & .013 & -.069 & -.041 & .91 \\
Multiple Faces & .060 & -.127 & .097 & .038 & -.051 & -.033 & .096 \\
Stock Photos & 0.043 & & 0.64 & .052 & .021 & -.34 & \\
\hline Image & gender & $\mathrm{Age}$ & $\mathrm{Ex}$ & $\mathrm{Ag}$ & $\mathrm{Op}$ & $\mathrm{Ne}$ & $\mathrm{Co}$
\end{tabular}

Demographics

\begin{tabular}{cccccccc}
\hline gender & 1 & .196 & -.188 & .083 & -.015 & .118 & .063 \\
Age & .196 & 1 & -.185 & -.190 & -.033 & .137 & .005 \\
Asian & .069 & -.049 & -.200 & -.139 & -.064 & .053 & -.010 \\
Black & .106 & -.077 & -.148 & -.147 & -.003 & -.015 & -.018 \\
White & .196 & 1.000 & .185 & -.190 & -.033 & .137 & .005 \\
Indian & -.243 & .201 & .226 & -.164 & -.073 & .000 & -.013 \\
\hline Facial & gander & Age & $\mathrm{Ex}$ & $\mathrm{Ag}$ & $\mathrm{Op}$ & $\mathrm{Ne}$ & $\mathrm{Co}$ \\
Presentation & & & & & & & \\
\hline No Glasses & -.094 & -.060 & .015 & .587 & -.133 & -.393 & .255
\end{tabular}




\begin{tabular}{|c|c|c|c|c|c|c|c|}
\hline Reading & .020 & .052 & -.130 & .097 & .018 & .033 & -.065 \\
\hline \multicolumn{8}{|l|}{ Glasses } \\
\hline Sunglasses & -.037 & .067 & .020 & -.033 & -.045 & -.076 & -.046 \\
\hline Yaw Angle & .049 & -.011 & .046 & -.024 & .045 & -.068 & -.081 \\
\hline Pitch Angle & -.190 & -.144 & .061 & -.098 & .043 & -.028 & .072 \\
\hline Roll Angle & -.003 & .029 & -.008 & -.013 & -.027 & .031 & .045 \\
\hline Facial & gender & Age & Ex & $\mathrm{Ag}$ & Op & $\mathrm{Ne}$ & Co \\
\hline \multicolumn{8}{|l|}{ Expressions } \\
\hline Happy & -.188 & .185 & 1 & .727 & -.191 & -.183 & .191 \\
\hline Neutral & .083 & -.190 & -.727 & 1 & .137 & -.166 & .116 \\
\hline Surprise & -.015 & -.033 & -.191 & -.137 & 1 & -.051 & .022 \\
\hline Sadness & .063 & .005 & -.191 & -.116 & .022 & .028 & -.010 \\
\hline Disgust & .118 & .137 & -.183 & -.166 & .051 & 1 & -.028 \\
\hline Anger & .069 & -.049 & -.200 & -.139 & .064 & .053 & 1 \\
\hline Fear & .106 & -.077 & -.148 & -.147 & .003 & -.015 & .018 \\
\hline Smiling Degree & -.057 & .062 & .056 & .130 & -.020 & -.014 & .018 \\
\hline
\end{tabular}

\section{The proposed face detection algorithm}

The proposed face detection algorithm contains two major modules: (1) face localization for finding face candidates; and (2) facial feature detection for verifying detected face candidates. The algorithm first detects skin regions that possibly contain a human face. The skin detection algorithm is based on statistical image processing using Face++ API.

Extraversion. Extraversion is a trait mainly involves interaction with the outside world. These types of users are correlated the highest out of all traits with colorful images. Their photos do not have any correlation with the color attributes that make a photo aesthetically, except for positive correlation with sharpness. Extroverts are correlated only with the use of the rule of thirds among the other composition features.

Extraversion is mostly related to the number of faces of the pictures, Similar to Conscientiousness. However, Extraverts slightly prefer images with more people, which caused a small face ratio. Extraversion, however unlike other personalities, 
which negatively correlated with the age of the presenting faces, so the users either appear younger in their profiles or photographed with other young(er) people.

Extroverted people are also strongly associated with not displaying reading glasses. For facial expressions, Extraverts and have almost similar emotions as Agreeableness users.

Agreeable. The agreeableness trait is characterized by social harmony and cooperation while, users tend to have pictures with lots of faces, characterized by a strong level of warm color, and a positive association with the rule of thirds. Moreover, disagreeable ones tend to have pictures where they are portrayed alone as well as using stock photos that are having pictures of pets, food and travels. Above all, it seems they do not choose their pictures carefully, as most of them are shot out or too bright.

According to psychology theory, Facial emotion patterns are a strong correlation with smiling, happiness and positive emotion and low in negative emotion expressions. Intriguingly, this is different from Conscientious people who are high in positive facial emotions and express this through the overall color tone of the image similarly to agreeable people.

Openness. The personality dimension of Openness can be correlated sub-traits of Intellect, which reflect intellectual, and Openness to Experience that indicates engagement with perceptual and aesthetic domains, as mentioned in (De Young, et al. 2007). Our analysis reveals that the users are most likely to have profile pictures other than faces.

It is quite obvious that users with high Openness are notably correlated to the majority of features indicative of the better aesthetic quality of their photos. In general, the images are high in contrast, sharpness, saturation, and less blur. However, their photos are less colorful. Naturalness is anti-correlated perhaps because of the artistic quality of images and the grayscale picture.

For facial presentation, the results indicate the use of reading glasses, but not sunglasses and the picture appear large when it contains a face in it. According to (Hellstrom, Tekle, 1994) psychology research has shown that a person wearing reading glasses is more intelligent or has intellectual virtues. 
Facial emotions are related to the use of color effect (Wexner, 1954). Photos of Openness users are higher in negative emotions, particularly sadness, disgust and anger, and lower inattention, smiling and positive emotion, especially happiness.

Neuroticism. Neuroticism is related to negative emotions and emotional instability. It is the opposite of Extraversion and Agreeableness trait. Notably, photos of neurotic people are perhaps anti-correlated with colorfulness. The average color emotion correlations are with negative color emotions. In terms of composition, neurotic people show simple images without considering the rule of thirds. Although this is similar for Openness, the photos of Neurotic people do not show the aesthetic features that characterize openness do.

Neurotic people do not like to present faces. In a case that a face is present, then it is mainly with reading glasses. Presence of reading glasses has been associated with perceived introversion and a decrease in attractiveness. Concerning facial emotions, Neuroticism displays, as expected, Lack of emotion expressions in profile picture thus the characteristics of the Neuroticism trait.

Conscientiousness. Conscientiousness is the personality trait related to orderliness, planned behavior and self- discipline. The images indicate that profile images with faces, especially with only one face, which indicators of higher Conscientiousness. So, the users high in this trait prefer the expected behavior like posting a picture of themselves.

In terms of colors, Conscientious users prefer pictures that are natural and bright, not grayscale and overall colorful without considering the rule of thirds. Despite this, their pictures are not more aesthetic, being anti-correlated with sharpness and positively correlated with blur. Conscientious user prefers showing the single face that might display pictures that make them seem older and without wearing any glasses.

Facial expressions are indicative of Conscientious people. The facial emotions of smiling and positive emotion like happiness are positively correlated, while negative mood, like disgust, anger and sadness, are anti-correlated. In general, Conscientious people express the most emotions across all five traits after 
the Openness and Agreeable users. This does not match with Conscientious people but is explainable by taking into account that in a profile picture, a person is expected to smile and appear happy.

\section{Results and Discussion}

OSNs integrate various sources of personal information that mirror those found in private thoughts, personal environments, social behavior and facialimages, all of which are known to contain valid information about personality (Ambady, Skowronski, 2008; Vazire, Gosling, 2004). In other words, the profile photos on social media and personality traits are related. Accordingly, the extended real-life hypothesis predicts that people use OSNs to communicate their real personality.

Facial expressions give user personality and the intent of the image. The viewers can judge a simple change in the expression of the face. Facial features include the type of glasses wore by the face that is, whether sunglasses or reading glasses, whether the beard is present or not (Hellstrom, Tekle, 1994).

The paper managed to answer the mean question that personality trait can be inferred from the twitter's user profile picture. The study computed different visual features for (500) profile images in the classification of the Big Five personality traits, to predict emotions and demographic profiles from user's chose profile picture and several exciting insights have emerged, which pave the way for future work.

In a nutshell, a remarked message by the current paper is that people are sharing more information than what they think unconsciously through social networks. In other words, any simple action, like uploading a profile picture on Twitter or other social platforms, may unveil some aspects of our personality, and this in turns may activate several possible business applications.

The study's results represent a first look at the accuracy of individuals' self-portrayals on OSNs and revealed that each personality trait had a type of profile picture posting on social media. Users with Extraversion, Agreeableness and Conscientiousness tend to post more photos that contain one or more faces. Users with Neuroticism and Openness tend 
to post-default images and pictures with no faces and with a negative mood. Extraversion and Conscientiousness users tend to post colorful images while Agreeableness, Openness and Neuroticism users post less colorful images. Extrovert and stable users tend to have pictures where they are smiling, and they appear with other people. Neurotics tend to have images without humans and close-up faces. Agreeable persons tend to use profile pictures with grey color. Thus, this study represents a method for analyzing personality through social media profile picture choice.

\section{Conclusions and Recommendations for Future Study}

Twitter has changed and enhanced the way that people communicate with each other, with brands and companies, and with social movements and initiatives. With a recent study, Image is everything, as other Twitter user's first impression of an individual, and it can make a big difference in whether they decide to follow him/her.

The individual update stream is by far the most important part of the user's Twitter profile. However, the profile's presentation also needs to reflect something about the user and to put the best face forward.

Some of the best profile pages on Twitter are the ones that represent a user's personality. Someone who loves to ski might upload an image of his favorite mountain range as the profile background and pair it with an avatar that depicts him in ski goggles. There is no need to bare the user's soul, but individuals on Twitter want to know something about the user, and the public page is where they can first discover it.

This paper demonstrates an efficient approach to assess photo quality. The advanced aesthetic features are not only efficient but also discriminative. The experimental results show that using simple techniques is sufficient to reach great classification performance on different variability of photos.

The study noticed that there is a large variety of subjects depicted in the profile pictures, including symbols neutral scenes and animals, butterflies, and stock photos (non-facial stimuli, 
e.g. cars, planes or natural scenes). In the future, it would be interesting to test artistic figures profiles and models extracted from more homogeneous dataset, for example, selecting only profile pictures containing faces.

As discussed, automatic personality assessment may open up new avenues for developing products and services. Such as, testing person posture and the colors of the costumes that the individual is wearing which can reveal a lot about the personality in the future. At the same time, privacy concerns and ethical challenges may also arise from the capacity to identify individuals' psychological traits from their liked images.

In addition, it is important to suit the picture to the platform, with an understanding of the prevailing code of behavior. For example, not to post a swimsuit photo on LinkedIn or a lectern shot on Twitter. The Twitter example may be less harmful than the LinkedIn example, but it still demonstrates poor social understanding, and thus, it still considered against the user. A picture with children or with family, for example, indicates an individual who is close to his/her family and who sees them as strong support. It also indicates that the critical thing in the individual's life is children and family. An "active" picture showing the user on a nature trip shows a desire to be considered etc. 


\section{References}

Alam, F., \& Riccardi, G. (2014). Predicting Personality Traits Using Multimodal Information. In Proceedings of the 2014 Multimedia, Workshop on Computational Personality Recognition, WCPR, 15-18.

Alexa.com. (2017). Top Sites. Retrieved from https://www.alexa.com/topsites

Ambady, N., \& Skowronski, J. (2008). First Impressions. New York: Guilford.

Back, M. D., Stopfer, J. M., Vazire, S., Gaddis, S., Schmukle, S. C., Egloff, B., \& Gosling, S. D. (2010). Facebook Profiles Reflect Actual Personality, not Self-Idealization. Psychological Science, 21, 372-374.

Borth, D., Ji, R., Chen, T., Breuel, T., \& Chang, S. F. (2013). Largescale Visual Sentiment Ontology and Detectors Using Adjective Noun Pairs. In Proceedings of the 21st ACM International Conference on Multimedia, MM, 223-232.

Bruce, V., \& Young, A. (2012). Chapter 6: Face Perception. New York: Psychology Press, 253-313.

Burger, J. D., Henderson, J., Kim, G., \& Zarrella, G. (2011). Discriminating Gender on Twitter. In Proceedings of the Conference on Empirical Methods in Natural Language Processing, 1301-1309. Association for Computational Linguistics.

Celli, F., Bruni, E., \& Lepri, B. (2014). Automatic Personality and Interaction Style Recognition from Facebook Profile Pictures, Proceedings of the ACM International Conference on Multimedia, 1101-1104.

Correa, T., Hinsley, A.W., \& Zuniga, H.G. (2010). Who Interacts on the Web? The Interaction of User's Personality and Social Media Use, Computers in Human Behavior, 26(2), 247-253.

Counts, S., \& Stecher, K. (2009). Self-Presentation of Personality During Online Profile Creation. In ICWSM, 191-194.

Danah, B., Golder, S., \& Lotan, G. (2010). Tweet, Tweet, Retweet: Conversational Aspects of Retweeting on Twitter. Hawaii International Conference on System Sciences, 1-10. 2

Datta, R., Joshi, D., Li, J., \& Wang, J. Z. (2006). Studying 
Aesthetics in Photographic Images Using a Computational Approach. In Proceedings of the 9th European Conference on Computer Vision, ECCV, 288-301.

De Young, C. G., Quilty, L. C., \& Peterson, J. B. (2007). Between Facets and Domains: 10 Aspects of the Big five. Journal of Personality and Social Psychology. 93(5), 10.1037/00223514.93.5.880, 880-896.

Ekman, P., \& Friesen, W. V. (1971). Constants Across Cultures in the Face and Emotion", Journal of Personality and Social Psychology, 17(2), 124-129.

Golbeck, J. (2005). Computing and Applying Trust in WebBased Social Networks. PhD Thesis, University of Maryland, College Park.

Gong, Shaogang, Stephen, J. McKenna, \& Alexandra Psarrou. (2000). Dynamic Vision: from Images to Face Recognition. Imperial College Press: London, 14 (6), 619-621.

Gonzales, A. L., \& Hancock, J. T. (2008). Identity Shift in Computer-Mediated Environments. Media Psychology, 11, 167185.

Halder, R., Sengupta, S., Pal, A., Ghosh, S., \& Kundu, D. (2016) Real Time Facial Emotion Recognition Based on Image Processing and Machine Learning, International Journal of Computer Applications, 139 (11).

Hall, J. A., Pennington, N., \& Lueders, A. (2013). Impression Management and Formation on Facebook: A Lens Model Approach. New Media \& Society 16(6), 958-982.

Haxby, J. V., Hoffman, E. A., \& Gobbini, M. I. (2000). The Distributed Human Neural System for Face Perception. Trends in Cognitive Sciences 4(6), 223-233.

Heide, B. V., D'Angelo, J. D., \& Schumaker, E. M. (2012). The Effects of Verbal Versus Photographic Self-Presentation on Impression Formation in Facebook. Journal of Communication 62(1), 98-116.

Hellstrom, A., \& Tekle, J. (1994). Person Perception Through Facial Photographs-Effects of Glasses, Hair, and Beard on Judgements of Occupation and Personal Qualities, European Journal of Social Psychology 24, 693-705. 
Huang, K., Wang, Q., \& Wu, Z. (2006). Natural Color Image Enhancement and Evaluation Algorithm Based on Human Visual System. Science Direct. Computer vision and image understanding 103(1), 52-63.

Huberman, B.A., Romero, D. M. \& Wu, F. (2009). Social Network that Matter: Twitter Under the Microscope. Peer-Reviewed Journal on the Internet, 14(1).

Jisun A.\& Ingmar W. (2016) \#greysanatomy versus \#yankees: Demographics and Hashtag Use on Twitter. Proceedings of the Twelfth International AAAI Conference on Web and Social Media.

John, O. P., Robins, R. W., \& Pervin, L. A. (2010). Handbook of Personality: Theory and Research. Guilford Press.

Kamenskaya, E., \& Kukharev, G. (2008). Recognition of Psychological Characteristics from Face. Metody Informatyki Stosowanej, 1(13), 59-73.

Kim, Y., Lee, H., \& Provost, E. M. (2013). Deep Learning for Robust Feature Generation in Audiovisual Emotion Recognition. In Proceedings of the IEEE International Conference on Acoustics, Speech and Signal Processing, ICASSP, 3687-3691.

Kim, E., Lee, J. A., Sung, Y., \& Choi, S. M. (2016). Predicting Selfie-Posting Behavior on Social Networking Sites: An Extension of Theory of Planned Behavior. Computers in Human Behavior, 62, 116-123.

Kosinski, M., Stillwell, D., \& Graepel, T. (2013). Private Traits and Attributes are Predictable from Digital Records of Human Behavior. Proceedings of the National Academy of Sciences of the United States of America, 110(15), 5802-5805.

Krämer, N. C., \& Winter, S. (2008). Impression Management 2.0: The Relationship of Self-Esteem, Extraversion, Self-Efficacy, and Self-Presentation Within Social Networking Sites. Journal of Media Psychology, 20(3),106- 116.

Kwak, H., Lee, C., Park, P., \& Moon, S. (2010). What is Twitter, a Social Network or a News Media? In International World Wide Web Conference, 591-600.

Lampos, V., \& Cristianini, N. (2012). Nowcasting Events from the Social Web with Statistical Learning. ACM Transactions on 
Intelligent Systems and Technology, 3(4).

Laura, F., Gruen, M. E., Poston, L., \& Dorsey, J. (2009). Twitter for Dummies. Wiley Publishing.

Liu, L., Preotiuc-Pietro, D., Samani, Z. R., Moghaddam, M. E., \& Ungar, L. (2016). Analyzing Personality Through Social Media Profile Picture Choice. In ICWSM, 211-220.

Lo, K.Y., Liu, K.H., \& Chen, C. S. (2012). Assessment of Photo Aesthetics with Efficiency, International Conference on Pattern Recognition. Proceedings of the National Academy of Sciences, 110(15), 5802-5805.

Manav, B. (2007). Color-Emotion Associations and Color Preferences: A case Study for Residences. Color Research Application. Psychology journal, 4 (9).

McCrae, R.R. (1989). Why I Advocate the Five-Factor Model: Joint Factor Analyses of the NEO-PI with Other Instruments. Personality Psychology: Recent Trends and Emerging Directions, 237-245.

McCrae, R. R., \& Costa, P. T. (2005). Personality in Adulthood: A Five-Factor Theory Perspective. The Guilford Press.

McCrae, R. R., \& John, O.P. (1992). An Introduction to the FiveFactor Model and its Applications, Journal of Personality 60,175215.

Naumann, L. P., Vazire, S., Rentfrow, P. J., \& Gosling, S. D. (2009). Personality Judgments Based on Physical Appearance. Personality and Social Psychology Bulletin 35(12),1661-1671.

Pantic, M. (2009). Machine Analysis of Facial Behavior: Naturalistic and Dynamic Behavior. Philosophical Transactions of the Royal Society B: Biological Sciences 364(1535), 3505-3513.

Peabody, D., \& Raad, B. D., (2002). The Substantive Nature of Psychological Personality Factors: A Comparison Across Languages. Journal of Personality and Social Psychology, 83(4), 983-997.

Penton-Voak, I. S., Pound, N., Little, A. C., \& Perrett, D. I. (2006). Personality Judgments from Natural and Composite Facial Images: More Evidence for a "Kernel of Truth" in Social Perception. Social Cognition 24(5), 607-640.॰

Rao, D., Yarowsky, D., Shreevats, A., \& Gupta, M. (2010). 
Classifying latent User Attributes in Twitter. In Proceedings of the 2nd International Workshop on Search and Mining UserGenerated Contents, 37-44. ACM.

Redi, M., Quercia, D., Graham, L. T., \& Gosling, S. D. (2015). Like Partying? Your Face Says It All. Predicting the Ambiance of Places with Profile Pictures. In Proceedings of the 9th International AAAI Conference on Weblogs and Social Media, ICWSM, 347-356.

Robert R. McCrae \& Oliver P. John. (1992). An Introduction to the Five-Factor Model and Its Applications. Journal of Personality 60(2), 175-215. doi: 10.1111/j.1467-6494.1992.tb00970.x.

Rosen, P. A., \& Kluemper, D. H. (2008). The Impact of the Big Five Personality Traits on the Acceptance of Social Networking Website. AMCIS 2008 Proceedings, 274.

Schmitt, D., Allik, J., McCrae, R., \& Benet, V. (2007). The Geographic Distribution of Big Five Personality Traits. Journal of Cross-Cultural Psychology, 38(2), 173-212.

Schrammel, J., Ko 'ffel, C., \& Tscheligi, M. (2009). Personality Traits, Usage Patterns and Information Disclosure in Online Communities. In BCS HCI '09: Proceedings of the 2009 British Computer Society Conference on Human-Computer Interaction, 169-174.

Schwartz, H. A., Eichstaedt, J. C., Kern, M. L., Dziurzynski, L., Ramones, S. M., Agrawal, M., Shah, A., Kosinski, M., Stillwell, D., Seligman, M. E., \& Ungar, L. H. (2013). Personality, Gender, and Age in the Language of Social Media: The Open-Vocabulary Approach. PloS ONE, 8(9).

Segalin, C., Celli, F., Polonio, L., Kosinski, M., Stillwell, D., Sebe, N., Cristani, M., \& Lepri, B. (2017). What Your Facebook Profile Picture Reveals About your Personality. Proceedings of the 25st ACM international conference on Multimedia.

Seger, C. A., \& Miller, E. K. (2010). Category Learning in the Brain. The Annual Reviews of Neuroscience. 10.1146? Andrew. neuro.051508.135546.

Selfhout, M., Burk, W., Branje, S., Denissen, J. van Aken, M. \& Meeus, W. (2010). Emerging Late Adolescent Friendship Networks and Big Five Personality Traits: A Social Network 
Approach. Journal of Personality, 78(2), 509-538.

Sharif, M., Mohsin, S., Hanan, R., Javed, M. Y., \& Raza, M. (2011). 3D Face Recognition Using Horizontal and Vertical Marked Strips, Sindh University Research Journal (SURJ), 43(1-A), 57-62. Soon-Gyo J., Jisun A., Haewoon K., Joni S., \& Bernard J. Jansen. (2018) Assessing the Accuracy of Four Popular Face Recognition Tools for Inferring Gender, Age, and Race, Proceedings of the Twelfth International AAAI Conference on Web and Social Media.

Vazire, S., \& Gosling, S.D. (2004). E-perceptions: Personality Impressions Based on Personal Websites. Journal of Personality and Social Psychology, 87(1), 123-132. 2

Verma, A., Koukuntla, K., Varma, R., \& Mukherjee, S. (2018). Automatic Assessment of Artistic Quality of Photos. Indian Institute of Information Technology Sri City.

Volkova, S., Bachrach, Y., \& Durme, B. (2016). Mining User Interests to Predict Perceived Psycho-Demographic Traits on Twitter. IEEE Second International Conference on, 36-43.

Wexner, L. B. (1954). The Degree to Which Colors (Hues) Are Associated with Mood-Tones, Journal of Applied Psychology 38(6), 432-435.

https://www.faceplusplus.com

You, Q., Luo, J., Jin, H., \& Yang, J. (2015). Robust Image Sentiment Analysis Using Progressively Trained and Domain Transferred Deep Networks. In the $29^{\text {th }}$ AAAI Conference on Artificial Intelligence, AAAI, 381-388.

*https://www.faceplusplus.com 
